\title{
Evaluation of Online Handwritten Characters for Penmanship Learning Support System
}

\author{
Tatsuya Yamaguchi ${ }^{1}$, Noriaki Muranaka ${ }^{2}$, and Masataka Tokumaru ${ }^{2}$ \\ ${ }^{1}$ Graduate School of Kansai University \\ 3-3-35 Yamate-cho, Suita-shi, Osaka 564-8680, Japan \\ ${ }^{2}$ Kansai University, 3-3-35 Yamate-cho, Suita-shi, Osaka 564-8680, Japan \\ $\{k 526329$, muranaka, toku\} @kansai-u.ac.jp
}

\begin{abstract}
This paper proposes a method for the evaluation of online handwritten characters for a penmanship learning support system. In Japan, many people desire to write beautiful characters, as evidenced by the fact that correspondence courses on penmanship are very popular. However, correspondence courses lack real-time feedback because of the time required for sending materials, namely, the learner's copy and the teacher's correct result. Therefore, we have developed a penmanship learning support system that automatically evaluates the characters that a learner writes using a personal digital assistant (PDA). We examined the validity of our proposed characterevaluation method, and proved it to be valid.
\end{abstract}

Keywords: Penmanship, Character Evaluation, PDA, Similarity, Balance, Speed, Advice.

\section{Introduction}

In recent years, many people have begun to use the personal computer (PC) in their daily lives, because its performance has improved and its price has declined. Therefore, various learning support systems that use the PC have been studied $[1,2]$. Among these studies, support systems that promote the learning of calligraphy and penmanship have been actively studied $[3,4]$. One reason for such interest is that there are many people who desire to write beautiful characters. In Japan, it is said that handwritten characters indicate one's personality. However, many people do not write characters frequently because of the diffusion of PCs and mobile phones, although the Japanese still write important documents, letters, and resumes by hand to communicate their feelings more precisely. Therefore, many people are learning penmanship. The methods for learning penmanship include correspondence courses and classes. In correspondence courses, learners learn the shape and balance of a character on the basis of a model character in the text and its accompanying explanation, at their convenience. Then, the learner sends his/her copy of the character to a teacher, and the teacher corrects it and sends the result back to the learner. Thus, correspondence courses lack real-time feedback because of the time 
required for sending materials. Attending a classroom course is the best way to learn penmanship because of the direct interaction between teachers and learners. However, because learners must attend penmanship classes regularly, classroom courses are not suitable for busy people. Therefore, we have developed a penmanship learning support system that automatically evaluates the characters a learner writes using a personal digital assistant (PDA) or a liquid-crystal pen display. The purpose of this system is to reproduce the environment of a penmanship classroom at home. Furthermore, learners can learn penmanship using a palm-sized PDA without being restricted for place or time. This system evaluates the characters a learner writes by comparing the features of model characters with those of the characters written by the learner. By evaluating these features, learners begin considering the features of the characters. We had proposed a feature-extraction method in a previous work [5]. By extracting the features on the system, we can apply one character-evaluation method to all characters. In other words, it is not necessary to prepare a separate characterevaluation method for each character. This paper describes the features of this character-evaluation method.

\section{System Overview}

This system uses two types of characters: model characters and learner's characters. Model characters are the characters a penmanship teacher has written beforehand. Learner's characters are those a learner writes when he/she learns. Learners learn the shape and balance of model characters by copying them. The learning processes in the system are similar to classroom techniques of penmanship. First, learners practice by tracing a model character. Next, they make a fair copy of it. Then, the system evaluates their character and presents advice. The learners review the advice and repeat this process.

\subsection{Hardware}

The authors implemented this system on a PDA (Fig. 1). The PDA features a palmsize coordinate input device that provides easy synchronization between a PC and the PDA.

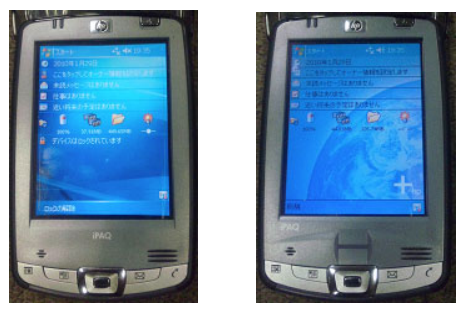

Fig. 1. Left: HP iPAQ hx2400, Right: HP iPAQ hx2700 


\subsection{Character Data}

The character data has coordinates at intervals of $10 \mathrm{~ms}$, an example of which is shown in Fig. 2(b). These intervals enable the reproduction of the writing process (the movement of the pen point) of the character. The data text consists of the index, takeoff and landing of the pen point (take-off: 0, landing: 1), stroke count, abscissa, and ordinate (from the left). The index is the time elapsed between reaching the coordinates and starting to write a character.

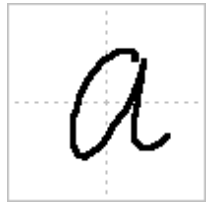

(a)

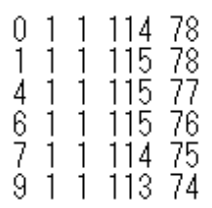

(b)

Fig. 2. For a character "a" written as (a), the system saves a data text of the character; (b) shows a part of the data text

\subsection{Function}

This system has four types of functions, namely, animation, trace, evaluation, and advice.

The animation function reproduces the writing process of a character that a teacher and learner write. This is the biggest advantage of device learning because paper learning tends to lose this process. Furthermore, this function enables the determination of the speed at which a character is written.

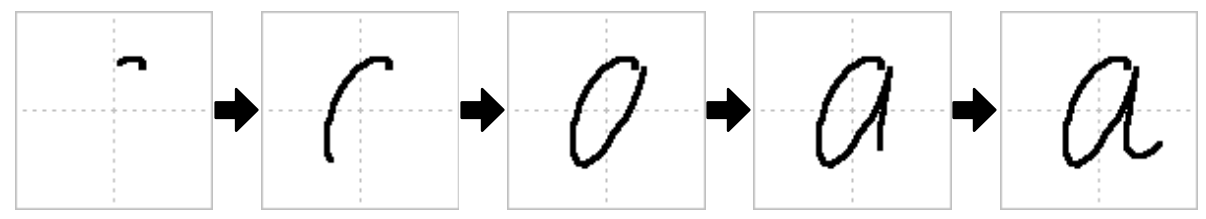

Fig. 3. Reproduction image of the animation function

The trace function of this system enables the tracing of the model character. In correspondence courses and classes on penmanship, a learner practices initially by tracing model characters.

The evaluation function evaluates the learner's character on a scale of 0 to 100 . The elements of evaluation include similarity, balance, and speed of the character. This paper describes the details of the evaluation method in Section 3.

The presentation of advice is divided into two categories: an accompanying explanation and a correction. Accompanying explanation describes how to write a beautiful character before you write it. Correction teaches what needs to be corrected 
in the character after you write it. This system uses this advice, and consequently learners progress their penmanship learning. In this paper, the advice function indicates a correction.

\subsection{GUI}

The GUI in this system contains a learning window, a result window, and an advice window for each evaluation element.

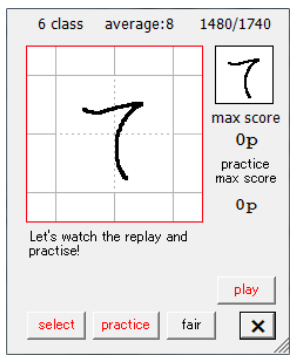

(a)

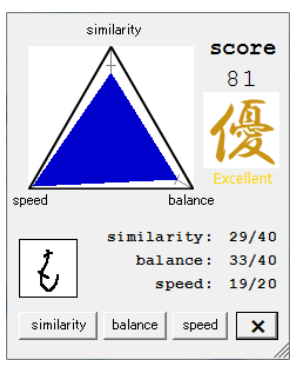

(e)

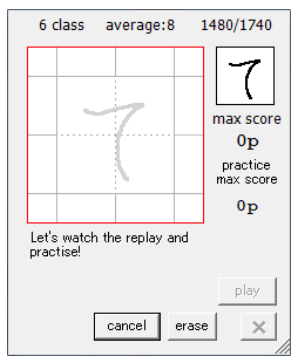

(b)

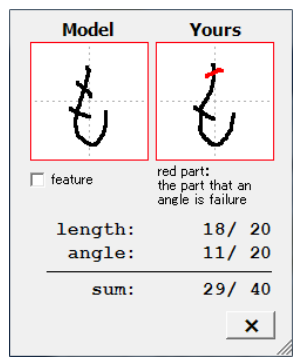

(f)

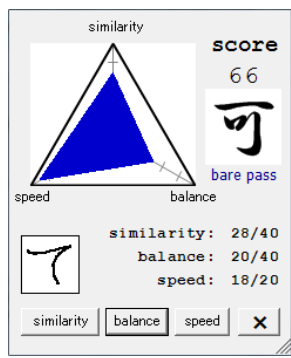

(c)

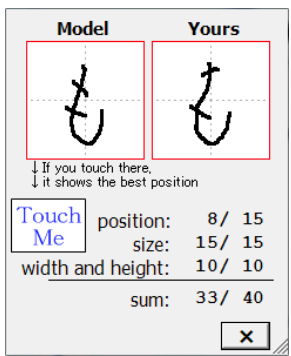

(g)

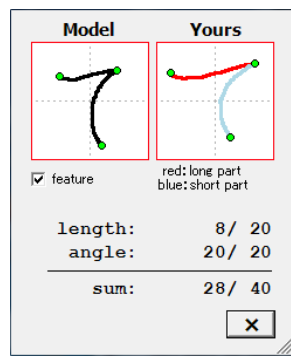

(d)

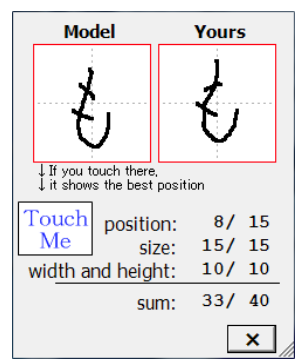

(h)

Fig. 4. Windows of the penmanship learning support system. (a) Learning window. The character in the upper-right corner of the window is a model character. In this window, learners can reproduce the writing process, practice, and make a fair copy of a model character. (b) Practice window. In this window, learners can trace the model character. (c) and (e) Result windows. (d) and (f) Advice windows regarding similarity. (g) and (h) Advice windows regarding balance. (g) is the normal window. When a learner touches "Touch Me" on (g), the window becomes $(\mathrm{h})$, which shows the best position of each stroke.

\section{Character Evaluation}

This system evaluates a handwritten character on a scale of 0 to 100 on the basis of the evaluation elements of similarity, balance, and speed. Fig. 5 shows the itemized evaluation elements and their maximum scores. 


total $(100 p)\left\{\begin{array}{ccc}\text { similarity } & (40 p) & \left\{\begin{array}{cc}\text { length } & (20 p) \\ \text { angle } & (20 p)\end{array}\right. \\ \text { balance } & (40 p)\left\{\begin{array}{cc}\text { position } & (15 p) \\ \text { size } & (15 p) \\ \text { widthand height } & (10 p)\end{array}\right. \\ \text { speed } & (20 p) & \left\{\begin{array}{cc}\text { speed rate } & (10 p) \\ \text { speed } & (10 p)\end{array}\right.\end{array}\right.$

Fig. 5. Allotment of points. Values in parentheses are the maximum scores. The total score is 100 points

\subsection{Similarity}

Similarity is evaluated on the basis of the lengths and angles between the features of a character. The feature-extraction method is described in [5]. After the system extracts the features of each character, it maps the features of the model character and learner's character (Fig. 6). The mapping method is described in [6]. The length and angle are evaluated from the lengths and angles between the mapped features in a row using Table 1 and the following equations:

$$
\begin{aligned}
& L r_{i}=\sqrt{30 / L m_{i}}, \\
& A r_{i}=\sqrt{L m_{i} \times L l_{i} / \sum_{i=1}^{n} L m_{i}}, \\
& L_{\text {ave }}=\frac{1}{n} \log _{2}\left[\sum_{i=1}^{n} L l_{i} / \sum_{i=1}^{n} L m_{i}\right] \text {, } \\
& L d_{i}=\left[L r_{i} \times\left(L m_{i}-L l_{i} / L_{a v e}\right)\right]^{2}, \\
& A d_{i}=\left[A r_{i} \times\left(A m_{i}-A l_{i}\right)\right]^{2}, \\
& L d=\frac{1}{n} \sum_{i=1}^{n}\left\{\begin{array}{cc}
\left(L d_{i}+20\right) / 5 & \left(L d_{i}>5\right) \\
L d_{i} & (\text { otherwise })
\end{array},\right. \\
& A d=\frac{1}{n} \sum_{i=1}^{n}\left\{\begin{array}{cc}
\left(A d_{i}+4\right) / 5 & \left(A d_{i}>1\right) \\
A d_{i} & \text { (otherwise) }
\end{array} .\right.
\end{aligned}
$$

In Table 1 and equations (1)-(7), $L$ : length, $A$ : angle, $m$ : model character, $l$ : learner's character, $r$ : ratio of the model character and the learner's character, $d$ : 
difference, $i$ : index, and $n$ : total section number. The scores of the length and angle are given by the points deducted, depending on the solution of (6) and (7), from 20.

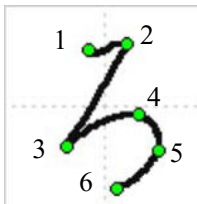

(a)

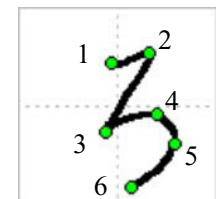

(b)

Fig. 6. Mapping points of the features in each character. (a) Model character. (b) Learner's character. Circles denote the features of each character.

Table 1. Length and angle of the features mapped in Fig. 6. Section is the area between the character's features, Model is the length and angle in a section of the model character, and Learner's is the length and angle of the learner's character.
(a) Length
(b) Angle

\begin{tabular}{|r|r|r|r|}
\hline $\begin{array}{c}\text { Index } \\
(i)\end{array}$ & Section & $\begin{array}{c}\text { Model } \\
\left(L m_{\mathrm{i}}\right)\end{array}$ & $\begin{array}{c}\text { Learner's } \\
\left(L l_{i}\right)\end{array}$ \\
\hline 1 & $1--2$ & 19.2 & 23.2 \\
\hline 2 & $2--3$ & 59.2 & 53.1 \\
\hline 3 & $3--4$ & 39.4 & 31.3 \\
\hline 4 & $4--5$ & 20.6 & 13.9 \\
\hline 5 & $5--6$ & 28.3 & 37.4 \\
\hline
\end{tabular}

\begin{tabular}{|r|r|r|r|}
\hline $\begin{array}{c}\text { Index } \\
(i)\end{array}$ & Section & $\begin{array}{c}\text { Model } \\
\left(\mathrm{Am} \mathrm{m}_{\mathrm{i}}\right)\end{array}$ & $\begin{array}{c}\text { Learner's } \\
\left(\mathrm{Al} \mathrm{l}_{i}\right)\end{array}$ \\
\hline 1 & $1--2$ & 9.0 & 10.3 \\
\hline 2 & $2--3$ & 239.5 & 235.4 \\
\hline 3 & $3--4$ & 24.0 & 19.7 \\
\hline 4 & $4--5$ & 299.1 & 324.0 \\
\hline 5 & $5--6$ & 222.1 & 235.9 \\
\hline
\end{tabular}

(Unit: pixel)

(Unit: degree)

\subsection{Balance}

The system evaluates the proportion of each stroke. Balance is evaluated on the basis of position, size, and width and height.

Position. The position of each stroke is the average of the $\mathrm{x}$ and $\mathrm{y}$ coordinates in the coordinate data that composes the stroke. It calculates the variance among the subtractions of the model's $x$ coordinates from those of the learner in each stroke. Similarly, the variance in the y coordinates is calculated. The score of position is given by the points deducted, depending on the square root of the sum of the variances in the $\mathrm{x}$ and $\mathrm{y}$ coordinates, from 15 .

Size. The stroke size is decided by the width and height in the stroke, as follows:

$$
\text { stroke size }=\left\{\begin{array}{lc}
\text { width }+ \text { height } / \text { width } & (\text { width }>\text { height }) \\
\text { height }+ \text { width } / \text { height } & (\text { otherwise })
\end{array} .\right.
$$

The score of the size is given by the points deducted, depending on the variance of the stroke sizes, from 15. 
Width and Height. $\sigma_{W}$ and $\sigma_{H}$ are calculated from the following equations:

$$
\begin{gathered}
\text { ave }=\frac{1}{m} \sum_{i=1}^{m} \log _{2}\left(L_{i} / M_{i}\right), \\
\sigma=\frac{1}{m} \sum_{i=1}^{m}\left\{\left[\log _{2}\left(L_{i} / M_{i}\right)-a v e\right] \times\left|L_{i}-M_{i}\right| \times M_{i} / 50\right\}^{2} .
\end{gathered}
$$

In equations (9) and (10), $L_{i}$ : width and height in the $i$ th stroke of a learner's character, $M_{i}$ : width and height of the model character; and $m$ : total stroke number of the character. If $m=1, \sigma$ is calculated using the following equation:

$$
\sigma=\left|L_{i}-M_{i}\right| \times M_{i} / 50
$$

The scores of the width and height are given by the points deducted, depending on $P$, from 10. $P$ is calculated using the following equation:

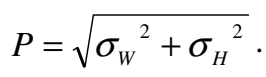

\subsection{Speed}

The system evaluates whether a character is written with a well-modulated speed. Speed is evaluated on the basis of the speed rate and the time rate.

Speed Rate. The speed rate is evaluated by the following evaluation method. First, it divides the model character into several parts at time intervals of $200 \mathrm{~ms}$. Next, it divides the learner's characters into part numbers of the model character. Then, it calculates the speed in each section of the characters and the relative ratio of each section speed of the model character and the corresponding section speed of the learner's character. The score of the speed rate is the number proportional to the sample variance of the relative ratio, subtracted from 15 . The following is a method for characters that consist of one stroke and a non-turn in the stroke. If these characteristics exist, the system divides the characters at those points. Then, it calculates the sample variance of the relative ratio in each section, and the weighted average. The weight is the sample size. In this case, the score of the speed rate is given by the points deducted, depending on the weighted average, from 10.

Time Rate. The time rate evaluates the relationship mentioned in the above section. The system calculates the time it takes to write each section of the model character and the learner's character, and the relative ratio of these characters in each section. The score of the time rate is given by the points deducted, depending on a sample variance of the relative ratio, from 10 . 


\section{Experiment and Results}

We examined whether the use of these evaluation elements in the system improved the efficiency of penmanship learning by testing it with ten university students. The examination spanned two weeks, for approximately 10 min per day. We provided PDAs that implement this system to the students, and used 46 Japanese "Hiragana" characters as model characters. We included a "Report button" in the system, so that a student could report a disagreement with the evaluation result by pushing the button. In reporting, a student selects a disagreed element among three evaluation elements (possible multiple-choice answers or non-choice answer). The evaluation result information is then saved in the PDA. After the examination period, we collected the PDAs and distributed a questionnaire (Table 2) to the students. Table 3 shows the experimental results.

Table 2. The questionnaire distributed to the students, with a scale of 1 to 5 : 1 . Strongly disagree, 2 . disagree, 3 . neither agree nor disagree, 4 . agree, 5 . strongly agree.

\begin{tabular}{l|l} 
Q. 1 & The evaluation method is valid. \\
Q. 2 & The model characters are beautiful. \\
Q. 3 & The more you use the system, the more you can write beautiful characters. \\
\hline
\end{tabular}

Table 3. Selected frequency for disagreement of each evaluation element (Similarity: S, Balance: B, Speed: S, and Non-choice: N), total report frequency (TRF), total learning frequency (TLF), and questionnaire (Table 2) results of the students

\begin{tabular}{r|rrrrrrrrr}
\hline Student & \multicolumn{1}{|c}{$\mathrm{S}$} & \multicolumn{1}{c}{$\mathrm{B}$} & \multicolumn{1}{c}{$\mathrm{S}$} & $\mathrm{N}$ & $\mathrm{TRF}$ & TLF & Q. 1 & Q. 2 & Q. 3 \\
\hline $\mathrm{A}$ & 3 & 2 & 12 & 3 & 16 & 1112 & 2 & 1 & 3 \\
$\mathrm{~B}$ & 1 & 0 & 1 & 0 & 2 & 198 & 4 & 5 & 4 \\
$\mathrm{C}$ & 0 & 0 & 0 & 0 & 0 & 180 & 5 & 4 & 4 \\
$\mathrm{D}$ & 0 & 0 & 1 & 0 & 1 & 392 & 4 & 4 & 4 \\
$\mathrm{E}$ & 0 & 0 & 4 & 0 & 4 & 701 & 4 & 3 & 4 \\
$\mathrm{~F}$ & 0 & 0 & 1 & 0 & 1 & 960 & 2 & 3 & 4 \\
$\mathrm{G}$ & 0 & 0 & 0 & 0 & 0 & 2226 & 5 & 5 & 4 \\
$\mathrm{H}$ & 0 & 0 & 0 & 0 & 0 & 368 & 2 & 2 & 3 \\
$\mathrm{I}$ & 1 & 1 & 1 & 2 & 4 & 179 & 4 & 5 & 4 \\
$\mathrm{~J}$ & 9 & 16 & 17 & 0 & 22 & 151 & 2 & 2 & 3 \\
\hline Average & 1.4 & 1.9 & 3.7 & 0.5 & 5 & 647 & 3.4 & 3.4 & 3.7 \\
\hline
\end{tabular}

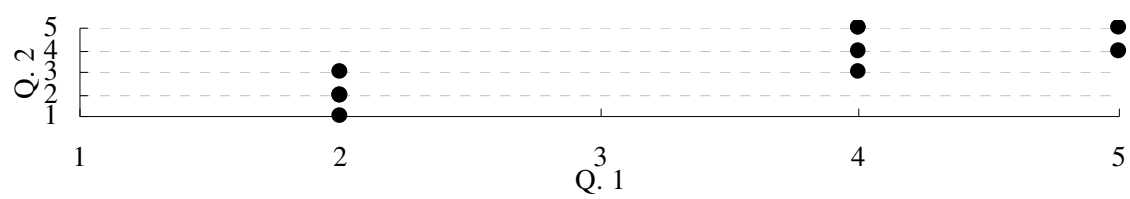

Fig. 7. Scatter diagram showing the questionnaire results of each student for Q. 1 and Q. 2 

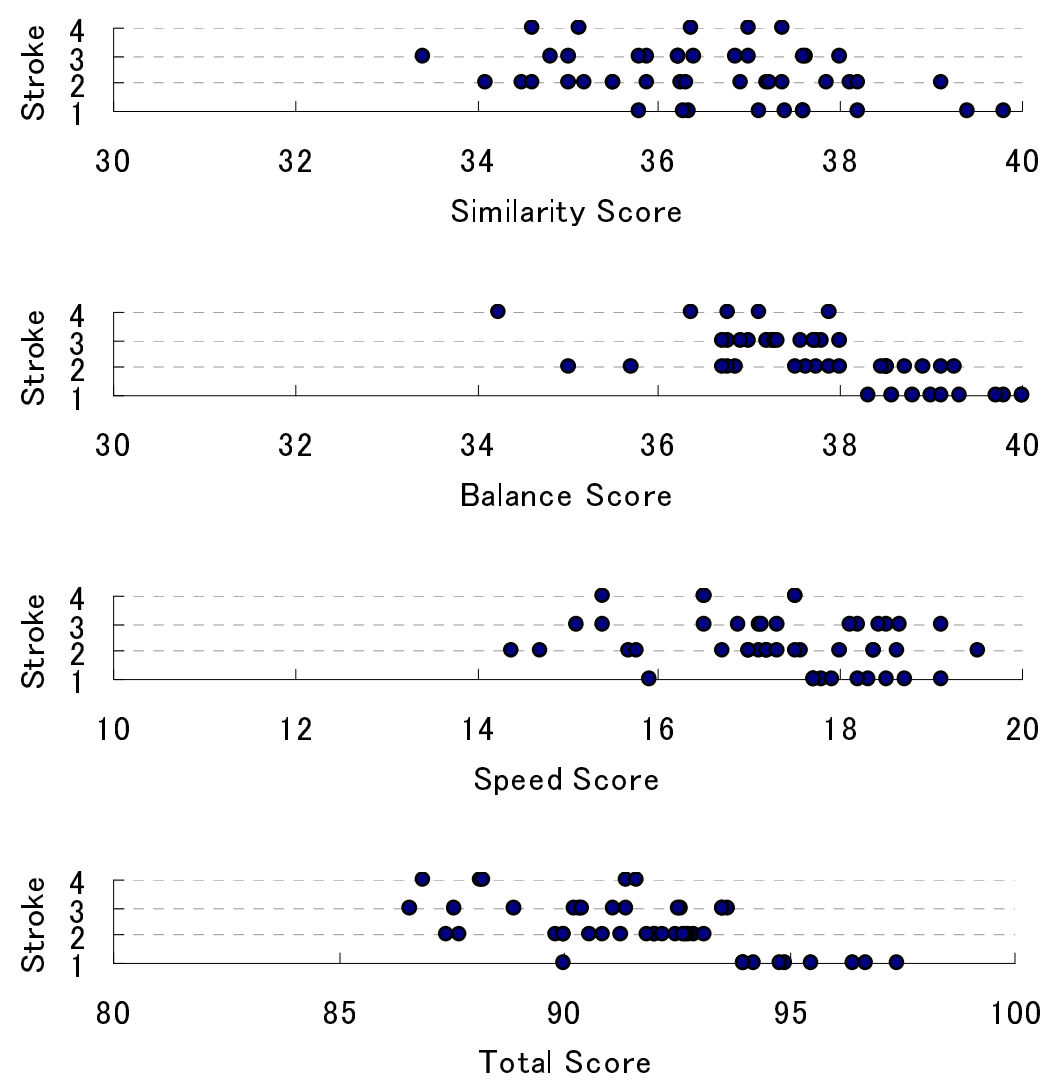

Fig. 8. Scatter diagrams showing the averaged evaluation results of each character in terms of the number of strokes, the averaged maximum score of each evaluation element, and total

\section{Discussion}

The evaluation method was proved valid because the total report frequency was 50, and seven out of ten students evaluated Q. 1 concerning validity as 4 or more (Table 3).

Speed was the most reported among the three evaluation elements because advice presentation regarding speed evaluation was provided to the students. In the evaluation of similarity and balance, the system indicates the incorrect part in color. In terms of speed, it reproduces model characters and learner's characters at the same time. The system needs to provide better visual advice.

Fig. 7 shows the correlation between the validity of the evaluation method and the beauty of model characters. It indicates that validity is decided by whether a student likes the model character. There are many types of beautiful characters; therefore, the model character of the system should be a character the learner likes. 
Fig. 8 shows the relationship between the number of strokes in each character and the score of each evaluation element. The scores of the characters of one of the strokes are large, because these characters are the simplest. However, others show no correlation. This proves that the evaluation method is not dependent on the number of strokes in each character.

\section{Conclusion and Future Work}

We proposed a character-evaluation method and examined its validity. The method was proved valid because there were only a few student disagreements and seven out of ten students evaluated the questionnaire concerning validity as 4 or more. To obtain the agreement of more students, we need to provide visual advice of speed evaluation and prepare various model characters that the students like. In our future work, we propose providing visual advice and extending the system's experimentation with more people.

\section{References}

1. Miura, M., Kunifuji, S.: Hybrid approach of augmented classroom environment with digital pens and personal handhelds. In: Gabrys, B., Howlett, R.J., Jain, L.C. (eds.) KES 2006. LNCS (LNAI), vol. 4252, pp. 1019-1026. Springer, Heidelberg (2006)

2. Park, K.S., Leigh, J., Johnson, A.E., Carter, B., Brody, J., Sosnoski, J.: Distance learning classroom using Virtual Harlem. In: 7th International Conference on Virtual Systems and Multimedia, Berkeley, pp. 489-498 (2001)

3. Ichimura, H., Suzuki, M., Murai, M., Yamashita, S., Kuroda, M., Kuroiwa, T., Yoshida, K., Yazawa, S., Mizuno, T., Sakai, S.: Design of Next Generation Distance-Learning System for Penmanship and Calligraphy. ACIS International Journal of Computer and Information Science 2(4), 162-171 (2001)

4. Yamasaki, T., Hattori, T.: Computer Calligraphy: Brush Written Kanji Formation Based on the Calligraphic Skill Knowledge. IEICE Transactions on Information and Systems E80-D 2, 17-175 (1997)

5. Yamaguchi, T., Muranaka, N., Tokumaru, M.: Penmanship Learning Support System: Feature Extraction for Online Handwritten Characters. In: 9th International Conference on Entertainment Computing, Entertainment Computing - ICEC2010, Seoul, pp. 496-498 (2010)

6. Sawamoto, T., Muranaka, N., Tokumaru, M., Imanishi, S.: Construction of the penmanship learning support system-Examination about the features extraction and mapping during model character and handwriting character. IEICE Tech. Rep., 109(335), 89-94 (2009) (in Japanese) 